\title{
Monogenic Functions of Higher Spin
}

\author{
F. Sommen
}

\begin{abstract}
We present a definition for monogenic functions of higher spin and establish the Fischer decomposition with respect to this notion.
\end{abstract}

Keywords: Clifford analysis, Dirac operators, spin representations

AMS subject classification: $30 \mathrm{G} 35$

\section{Introduction}

Let $R_{m}$ be the Clifford algebra over the Euclidean space $\mathbb{R}^{m}$ with basis $\left\{e_{j}\right\}_{1 \leq j \leq m}$ and defining relations $e_{j} e_{k}+e_{k} e_{j}=-2 \delta_{j k}(1 \leq j, k \leq m)$ where $\delta_{j k}$ is the Kronecker symbol. Then the Dirac operator $\partial$ is given by $\partial_{\underline{x}}=\sum_{j=1}^{m} e_{j} \partial_{x_{j}}\left(\underline{x}=\sum_{j=1}^{m} x_{j} e_{j}\right)$ and monogenic functions are $R_{m}$-valued solutions of the equation $\partial_{\underline{x}} f(\underline{x})=0$. Monogenic functions of this type may transform under the spin group. $\operatorname{Spin}(m)$ in two different ways. First note that $\operatorname{Spin}(\mathrm{m})$ is a subgroup of $R_{m}$ consisting of elements of the form $s=\underline{w}_{1} \cdots \underline{w}_{2 k}$ whereby $\underline{w}_{j} \in \mathbb{R}^{m}(j=1, \ldots, 2 k)$ are unit vectors (i.e. $\left.\underline{w}_{j}^{2}=-1\right)$. Next let $a \rightarrow \bar{a}$ be the main anti-involution on $\dot{R}_{m}$ determined by $\overline{a b}=\bar{b} \bar{a}$ and $\bar{e}_{j}=-e_{j}$. Then we may consider the two representations

$$
L(s) f(\underline{x})=s f(\bar{s} \underline{x} s) \quad \text { and } \quad H(s) f(\underline{x})=s f(\bar{s} \underline{x} s) \bar{s}
$$

transforming monogenic functions into monogenic functions.

The first representation corresponds in, fact to fields with $\operatorname{spin} \frac{1}{2}$. Usually this representation is defined for spinor-valued functions; but spinor spaces may be seen as minimal left ideals of the real Clifford algebra $R_{m}$ (or in fact the complexified Clifford algebra $C_{m}$ which may be represented by spaces of the form $C_{m} I$ with $I$ being a primitive idempotent). The above definitions carry over to the complex or hyperbolic situation and in particular to the Minkowski space, where fields with spin $\frac{1}{2}$ correspond to the free electron field (see also [1]).

The second representation corresponds to fields with spin 1 . Note hereby that special examples of monogenic functions transforming in this way are functions with values in the space $R_{m, k}$ of real $k$-vectors. Monogenic functions like this may be interpreted as solutions to the Hodge system for harmonic forms. In particular, for $k=2$ and $m=4$ (Minkowski space) these functions correspond to the electromagnetic field (see, e.g., [3]).

F. Sommen: Univ. Gent, Dept. Math. Anal., Galglaan 2, 9000 Gent, Belgium 
But the above definition of monogenicity does not include functions with higher order spin. This is due to the fact that the Clifford algebra $R_{m}$ only contains the basic representations of the spin group $\operatorname{Spin}(\mathrm{m})$ which are the representation $l(s): a \rightarrow s a$ on spinor spaces and the representation $h(s): a \rightarrow s a \bar{s}$ on the spaces $R_{m, k}$ of $k$-vectors. To construct models for irreducible representations of the spin group $\operatorname{Spin}(\mathrm{m})$ with higher order weights one may use multilinear functions on $\mathbb{R}^{m}$ (or even on $R_{m}$ ) with values in $R_{m}$, called Clifford tensors. In our paper [4] we studied the algebra of Spin(m)-invariant operators on Clifford tensors while in [5] we introduced so called monogenic tensors thus leading to explicit models for all irreducible representations of the spin group Spin(m) (see also [2]). But due to the existence of an inner product on the Clifford algebra $R_{m}$, spaces of multilinear functions on $R_{m}$ may also be mapped isomorphically on $k$-fold tensor products $R_{m} \otimes \cdots \otimes R_{m}$ of $R_{m}$. Hence monogenic functions with higher order spin can be defined as function on $\mathbb{R}^{m}$ with values in $R_{m} \otimes \cdots \otimes R_{m}$. But there is even a better choice. The tensor product $R_{m} \otimes \cdots \otimes R_{m}$ itself, as a vector space, is isomorphic to a Clifford algebra $R_{m \cdot k}$ over $\mathbb{R}^{m \cdot k}$. This is the idea we use in this paper.

In Section 1 we give the definition of monogenic functions with values in $R_{m \cdot k}$ and we discuss the action of the spin group on them. In Section 2 we prove the Fischer decomposition for $R_{m \cdot k}$-valued homogeneous polynomials corresponding to this notion of monogenicity (further examples of Fischer decompositions related to this one may be found in $[6,9])$.

Different approaches to Dirac operators of higher spin were presented in $[2,7,8]$. In our approach no specific choice of an irreducible representation space is needed.

\section{Definition of monogenic functions of higher spin}

Let $\left\{e_{j, \ell}\right\}_{\substack{1 \leq j \leq m \\ 1 \leq \ell \leq k}}$ be an orthonormal basis of the space $\mathbb{R}^{m \cdot k}$ generating the Clifford algebra $R_{m \cdot k}$. Then for $\ell=1, \ldots, k$ we put $\partial_{\underline{x_{\ell}}}=\sum_{j=1}^{m} e_{j, \ell} \partial_{x_{j}}$.

Definition 1. A function $f: \mathbb{R}^{m} \rightarrow R_{m \cdot k}$ is called monogenic of higher spin if it satisfies the system of equations $\partial_{\underline{x}} f(\underline{x})=0 \quad(\ell=1, \ldots, k)$.

Next, using the already abvailable Clifford algebra $R_{m}$ we may introduce embedding maps (.) $)_{\ell} R_{m} \rightarrow R_{m \cdot k}$ as follows. For $j=1, \ldots, m$ put $\left(e_{j}\right)_{\ell}=e_{j, \ell}$. This together with the property $(a b)_{\ell}=(a)_{\ell}(b)_{\ell}$ determines the map $(\cdot)_{\ell}$. In particular, for each $s^{\prime} \in$ $\operatorname{Spin}(m)$ we may consider the element $s_{\ell}=(s)_{\ell}$, thus leading to $k$ different realizations of the spin group $\operatorname{Spin}(\dot{\mathrm{m}})$ inside $R_{m \cdot k}$. tion

On functions $f: \mathbb{R}^{m} \rightarrow R_{m \cdot k}$ we may now consider the so called spin $\frac{k}{2}$-representa-

and we have the following

$$
L_{k}(s) f: f(\underline{x}) \rightarrow s_{1} \cdots s_{k} f(\bar{s} \underline{x} s)
$$

Theorem 1. For any function $f: \mathbb{R}^{m} \rightarrow R_{m \cdot k}$ which is monogenic of higher spin, the function $L_{k}(s) f$ is still monogenic of higher order spin.

Proof. It is sufficient to note that for $\ell \neq n$ and $s_{\ell} \in \operatorname{Spin}(m)$, the element $s_{\ell}$ commutes with the $n$-th Dirac operator $\partial_{\underline{x}_{n}}$ and that the elements $s_{\ell}(\ell=1, \ldots, m)$ are mutually commutative 
To make the link with functions with values in tensor products of $R_{m}$, note that the operators $\partial_{\underline{z}}$, are anti-commutative. Hence if we introduce new Clifford algebra elements $E_{1}, \ldots, E_{k}$, the operators $D_{\underline{x_{\ell}}}=\partial_{\underline{x_{\ell}}} E_{\ell}$ are mutually commutative and any function $f: \mathbb{R}^{m} \rightarrow R_{m \cdot k}$ satisfying the equations $\partial_{\underline{x}} f=0(l=1, \ldots, k)$ still satisfies the equations $D_{\underline{x}}, f=0(\ell=1, \ldots, k)$.

Note also that the algebra generated by the basis elements $e_{j, \ell} E_{\ell}(j=1 \ldots m, \ell=$ $1, \ldots, k)$ is isomorphic to the $k$-fold tensor product $R_{m} \otimes \cdots \otimes R_{m}$ of the Clifford algebra $R_{m}$. Hence by considering the functions $f$ with values in a somewhat larger Clifford algebra $R_{m \cdot k+k}$ one can incorporate tensor-valued as well as $R_{m \cdot k}$-valued functions.

\section{The Fischer decomposition}

We first introduce the Spin(m)-invariant Fischer inner product for homogeneous polynomials $R_{n}$ with values in $R_{m \cdot k}$. On $R_{m \cdot k}$ we consider the main anti-involution $a \rightarrow \bar{a}$ determined by $\bar{e}_{j, \ell}=-e_{j, \ell}(\ell=1, \ldots, k)$ and $\overline{a b}=\bar{b} \bar{a}$. Then the Fischer inner product is given by

$$
\left(R_{n}(\underline{x}), S_{n}(\underline{x})\right)=\bar{R}_{n}\left(\partial_{\underline{x}}\right) S_{n}(\underline{x}) \quad\left(\underline{x} \in \mathbb{R}^{m}\right) .
$$

It is readily seen that this inner product is invariant under $L_{k}$, i.e.

$$
\left(L_{k}(s) R_{n}, L_{k}(s) S_{n}\right)=\left(R_{n}, S_{n}\right)
$$

for all $s \in \operatorname{Spin}(m)$.

Next consider for $\underline{x} \in \mathbb{R}^{m}=R_{m, 1}$ the corresponding vector variables $(\underline{x})_{j}=$ $\underline{x}_{j}=\sum x_{\ell} e_{\ell, j}$ which are anti-commuting $R_{m \cdot k}$-valued functions satisfying $(\underline{x})_{j}^{2}=\underline{x}^{2}=$ $-\sum x_{\ell}^{2}(j=1, \ldots, k ; \ell=1, \ldots, m)$. Then we may consider the space of polynomials $R_{n}$ of the form

$$
R_{n}(x)=\sum \underline{x}_{j} R_{j, n-1}(x) \quad\left(\underline{x} \in \mathbb{R}^{m}\right),
$$

$R_{j, n-1}$ being homogeneous of degree $n-1$, and we have the following

Theorem 2 (Simple Fischer decomposition). Any homogeneous polynomial $R_{n}$ admits a unique orthogonal decomposition of the form

$$
R_{n}(x)=P_{n}(x)+\sum \underline{x}_{j} R_{j, n-1}(x)
$$

whereby $P_{n}$ is a homogeneous monogenic polynomial of higher.spin, i.e. $\partial_{\underline{x}_{1}} P_{n}=0$.

Proof. The theorem follows from the fact that the Fischer inner product is positive definite so that $R_{n}$ may always be decomposed as an orthogonal sum $R_{n}(x)=P_{n}(x)+$ $\sum \underline{x}_{j} R_{j, n-1}(x)$, and from the orthogonality and the definition of the Fischer inner product it follows that $P_{n}$ is monogenic

To arrive at a complete Fischer decomposition we consider the spaces $\mathcal{P}_{(n, j)}$ of homogeneous polynomials of degree $n$ and type $j$ to be defined recursively as follows : $\mathcal{P}_{(n, 0)}$ is the space of all homogeneous polynomials of degree $n$ while $\mathcal{P}_{(n, j)}$ is the subspace of $\mathcal{P}_{(n, j-1)}$ of polynomials of the form $\sum \underline{x}_{\ell} R_{\ell, n-1}(x)$ with $R_{\ell, n-1} \in \mathcal{P}_{(n-1, j-1)}$. We now come to 
Theorem 3 (Complete Fischer decomposition). Any polynomial $R_{(n, j)} \in \mathcal{P}_{(n, j)}$ admits a unique orthogonal decomposition of the form $R_{(n, j)}=P_{(n, j)}+R_{(n, j+1)}$ with $R_{(n, j+1)} \in \mathcal{P}_{(n, j+1)}$ and whereby $P_{(n, j)} \in \mathcal{P}_{(n, j)}$ is $(j+1)$-monogenic of higher spin, i.e. $\partial_{\underline{x}_{11}} \cdots \partial_{\underline{x}_{j+1}} P_{(n, j)}=0$.

Proof. The proof is similar to that of the previous theorem taking into account that the $j$-monogenicity condition is satisfied by any homogeneous polynomial of degree $k$ which is Fischer orthogonal to the space $\mathcal{P}_{(n, j+1)}$

By. recursive application of this theorem it follows that any homogeneous polynomial $R_{n}$ of degree $n$ admits a unique orthogonal decomposition of the form

$$
R_{n}=\sum_{j=0}^{n} P_{(n, j)}
$$

whereby $P_{(n, j)} \in \mathcal{P}_{(n, j)}$ is left $(j+1)$-monogenic of higher spin. This in fact establishes the canonical form of the Fischer decomposition. One can now look for characterizations of polynomials of the form $P_{(n, j)} \in \mathcal{P}_{(n, j)}$ which are left $(j+1)$-monogenic. They can be characterized in terms of solutions of special systems of equations similar to the monogenicity condition.

\section{References}

[1] Delanghe, R., Sommen, F. and V. Souček: Clifford Algebra and Spinor-Valued Functions: a Function Theory for the Dirac Operator (Mathematics and its Applications: Vol. 53). Dordrecht: Kluwer Acad. Publ. 1992.

[2] Gilbert, J. and M. Murray: Clifford Algebras and Dirac Operators in Harmonic Analysis. Cambridge: Cambridge Univ. Press 1990.

[3] Jancewicz, B.: Multivectors and Clifford Algebras in Electrodynamics. Singapore: World Sci. 1988.

[4] Sommen, F.: $S O(M)$-invariant operators on Clifford tensors. Fund. Theories Phys. 55 (1993), 193 - 202.

[5] Sommen, F.: Clifford tensor calculus. In: Proc. XXIIth Conf. Diff. Geom. Meth. Theor. Phys. (DGM), Ixtapa 1993 (eds.: J. Keller and Z. Oziewicz). Adv. Appl. Clifford Alg. (Proc. Suppl.), 4 S 1 (1994), 423 - 436.

[6] Sommen, F. and N. Van Acker: Functions of two vector variables. Adv. Appl. Clifford Alg. 4 (1994), $65-72$.

[7] Souček, V.: Clifford analysis for higher spin. Fund. Theories Phys. 55 (1993), 223 - 232.

[8] Stein, E. and G. Weiss: Generalization of the Cauchy-Riemann equations and representations of the rotation group. Amer. J. Math. 90 (1968), 163 - 196.

[9] Bernades, G., Sommen, F. and R. Delanghe: Fischer decomposition for two-sided monogenic functions. In preparation. 